\title{
Attention improves perceptual quality
}

\author{
Britt Anderson • Michael Druker
}

Published online: 2 October 2012

(C) Psychonomic Society, Inc. 2012

\begin{abstract}
Attention has been demonstrated to affect response speed, signal detection, and perceptual enhancement. We wished to determine whether exogenously cued attention had the additional effect of improving the veridicality of perceptual representations. We determined the accuracy and precision of orientation judgments for static and dynamic stimuli when they were, and when they were not, exogenously cued (luminance change). Valid exogenous cues led to more accurate responses that were also quicker. The attentional accuracy benefits were robust to stimulus degradation. Since our task displayed only one test stimulus at a time, response biases cannot account for our results. We concluded that exogenous cues can lead to percepts that are, on average, more similar to the external stimulus. Thus, exogenous cuing can improve perceptual quality.
\end{abstract}

Keywords Attention - Orientation · Exogenous cues · Coherent motion

To evaluate whether attention improves perception, one needs to show that perception is "better" with attention than without it. Demonstrations of attentional improvements have included shortened response times (RTs; Posner, Snyder, \& Davidson, 1980), improved signal detection (Moray, Fitter, Ostry, Favreau, \& Nagy, 1976), reduced noise (Dosher \& Lu, 2000b; Lee, Itti, Koch, \& Braun, 1999), and increases in signal intensity (Carrasco, Ling, \& Read, 2004).

Another sense in which attention might render perception "better" would be if it led to a more consistent, veridical

B. Anderson $(\triangle) \cdot$ M. Druker

Department of Psychology and Centre for Theoretical

Neuroscience, University of Waterloo,

200 University Avenue West,

Waterloo, Ontario N2L 1J6, Canada

e-mail: britt@uwaterloo.ca representation of a stimulus. Such a characterization of attentional improvement has been much less studied than other varieties. The most thorough treatment has been by Prinzmetal and colleagues (Prinzmetal, Amiri, Allen, \& Edwards, 1998; Prinzmetal, Nwachuku, Bodanski, Blumenfeld, \& Shimizu, 1997; Prinzmetal \& Wilson, 1997). They combined classic matching tasks with a second, dual task. Prinzmetal et al. (1998) reported that a simultaneously performed dual task made color, location, orientation, or spatial frequency matching more variable. Prinzmetal et al. (1997) found the same result for stimulus brightness and contrast. In Prinzmetal and Wilson (1997), the same effect was demonstrated for length estimation.

The studies of Prinzmetal et al. were conducted from the perspective of attention as a capacity-limited resource. By and large, they did not investigate spatial attention as manipulated by exogenous cues. In the two experiments that came closest to this, the results were mixed. In their Experiment 3, Prinzmetal et al. (1998) found an effect of attention on accuracy, but in Experiment 5 of Prinzmetal et al. (1997), in which an $80 \%$ valid location cue was used, the authors did not.

The present article serves two purposes. First, it evaluates whether noninformative luminance cues that exogenously cue attention to particular spatial locations can lead to "better" perceptual representations. By "better" we expand the usual considerations of attentional benefits to include "more veridical." This is measured by the deviation between the subject's report and the stimulus shown. If this deviation is smaller with attention than without it, perception is rendered more accurate. ${ }^{1}$ Second, our article presents a procedure that complements the classification task approach to studying attention. Classification tasks require a decision between alternatives, and this introduces the possibility of

\footnotetext{
${ }^{1}$ When attention is studied with classification tasks, the term accuracy refers to the proportion correct; that is a related, but distinct, use of the term.
} 
response biases (for an example of the interpretive challenges, see Anton-Erxleben, Abrams, \& Carrasco, 2011; Schneider, 2011); interpreting such data is further complicated by the correlation between response biases and response precision (Kerzel, Zarian, Gauch, \& Buetti, 2010). Since our matching procedure requires subjects to view only one stimulus at a time, response bias is not an issue. Also, our procedure yields a direct report of subjects' percepts, and thus requires few statistical assumptions; classification tasks infer perceptual representations from statistical curvefitting procedures and require assumptions about the probability distributions generating the errors.

\section{Method}

Figure 1 presents an overview of our experimental procedures.

\section{Subjects}

Our subjects were recruited from the undergraduate Research Experience Group at the University of Waterloo and were compensated with course credit. All experiments were approved by the University of Waterloo's Office of Research Ethics, and all subjects signed informed consent prior to beginning their research participation. Each experiment had 20 subjects, and each session was about 1 hour in length.

\section{General methods}

Subjects viewed stimuli on a computer and made responses using the computer keyboard. The experimental timings and display were programmed in Python using the PsychoPy library (Peirce, 2008). Subjects sat with their eyes at a distance approximately $60 \mathrm{~cm}$ from a CRT display of $33 \times$ $26.5 \mathrm{~cm}$, screen resolution of 1,280 $\times 1,024$ pixels, and that refreshed at $85 \mathrm{~Hz}$. Eye movements were not monitored, as stimuli were equally likely to be right or left of center and were too brief to be seen with a saccade. The subjects were instructed to look at the center of the screen throughout the experiments. Stimuli would appear $6 \mathrm{deg}$ to either the left or the right of fixation.

The experimental sessions consisted of 100 practice trials followed by five blocks of 150 trials each. The practice block familiarized subjects with the task and response methods. The display background was gray and had a measured luminance of $39.3 \mathrm{~cd} / \mathrm{m}^{2}$. A trial began with a white fixation cross (1.2 deg), followed by a white cue box $(4.4 \times 4.4 \mathrm{deg})$ appearing $6 \mathrm{deg}$ to the right or left of the fixation cross. The exogenous cue was a white box that surrounded one of the two possible target locations, was equally likely to be on the left or right, and was uninformative of target location (see Fig. 1). In the first set of experiments, $20 \%$ of the trials had

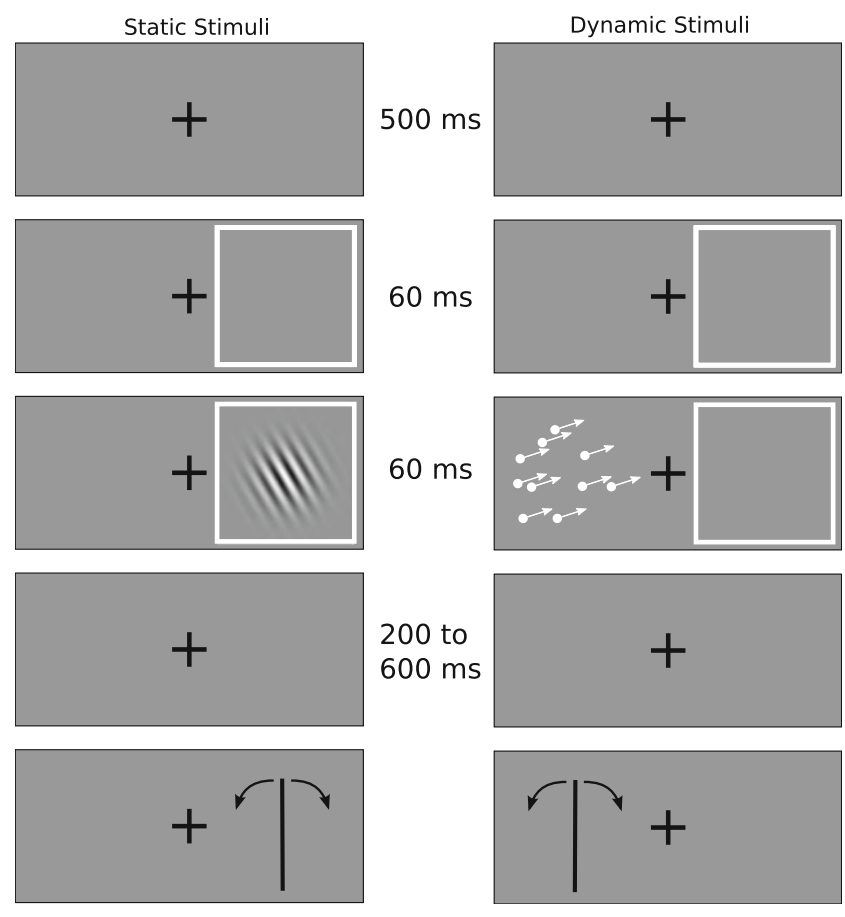

Fig. 1 General procedure. All experiments followed slight variants of this basic procedure: A fixation mark appeared and was followed by the abrupt onset of a white square, equally likely to appear to the left or right, and uninformative about the subsequent stimulus location. The stimulus, either an oriented static grating or a coherent moving-dot display, appeared briefly at either the left or right potential cue location. After the cue and stimulus were removed, a vertically oriented black line was presented at the location where the stimulus had been (the duration between stimulus removal and the line appearing was manipulated in two experiments). The subjects used the left and right arrow keys to orient the black line to match the orientation of the stimulus. When subjects were satisfied with their responses, they pressed the up arrow key to finalize it. On the left is a trial with a validly cued static grating stimulus, and the right side represents a trial with an invalidly cued dot-motion stimulus

"neutral" cues, in which the exogenous cue (the white box) appeared around both stimulus positions. When the cue appeared on the same side as the stimulus, the trial was designated as "valid," and the trial was "invalid" if the cue and stimulus were on opposite sides.

Static stimuli The static stimuli were sinusoidal gratings. A sine-wave texture of four cycles per degree of visual angle along a single dimension was displayed with a Gaussian mask (the $S D$ or space constant was $2 / 3$ of a degree). The patches were randomly rotated, and the contrast magnitude was $63 \%$ except in Experiment 3s, where contrast was a manipulated variable. In that experiment, we used a circular mask instead of the Gaussian mask to avoid confounding contrast with apparent stimulus size. The circular mask was $4 \mathrm{deg}$ in size.

Dynamic stimuli The dynamic stimuli were displays of coherently moving dots. The viewable field was a circle $4 \mathrm{deg}$ 
in size and composed of 20 dots. All of the dots moved coherently in a random, uniform direction, except in Experiment $3 \mathrm{~d}$, where the number of coherently moving dots was varied from two to 20, inclusive. The dots were live for the length of the display (five frames), and their size was four pixels wide. The dots moved at a rate of $0.15 \mathrm{deg} /$ frame $(12.75 \mathrm{deg} / \mathrm{s})$. For the experiment with decreased dot coherence, noise dots had the same speed, and each noise dot had a random but fixed direction. The dot motion was always upward and was uniformly chosen from 0 deg (right) to $180 \mathrm{deg}$ (left); $90 \mathrm{deg}$ was the vertical direction. We limited the dot motion range to $180 \mathrm{deg}$ to make it comparable to the static stimuli.

Stimulus timing The timings for the various experiments were consistent. The cue was presented for $118 \mathrm{~ms}$ total; it was presented for five frames $(59 \mathrm{~ms})$ alone, and then the stimulus would appear for five frames. The cue and stimulus offsets were simultaneous. The interval between cue and stimulus offset and the response meter onset was $200 \mathrm{~ms}$, except for the experiments on iconic decay, in which it could be either 200, 400, or $600 \mathrm{~ms}$.

Response recording and error calculation The response was visualized using a black line (4 deg long, about 0.07 deg thick) at the location where the stimulus had been presented. It always started out vertical, and subjects used the right and left arrow keys on a standard keyboard to tilt the line clockwise and counterclockwise, respectively. They pressed the up arrow key to indicate a complete response.

In Experiments 1s and 1d, when subjects held down the right or left arrow key, the speed of the rotation was $85 \mathrm{deg} / \mathrm{s}$ (geometric angle), which could be sped up to $255 \mathrm{deg} / \mathrm{s}$ by simultaneously holding the down arrow key. Few subjects found the latter function useful, so only one speed was used in the other experiments. That speed was set at a middle ground of $170 \mathrm{deg} / \mathrm{s}$ for Experiment 2s, but due to higher error rates, it was reverted to $85 \mathrm{deg} / \mathrm{s}$ for Experiments $2 \mathrm{~d}, 3 \mathrm{~s}$, and $3 \mathrm{~d}$. Tilt was always adjusted in whole degrees. The error was calculated as the minimum of the two angles that the response line created with the true orientation (static stimuli) or axis of motion (dynamic stimuli). This yielded errors in the range of $\pm 90 \mathrm{deg}$. The horizontal orientation was designated as $0 \mathrm{deg}$, and the vertical direction as +90 . Judgment errors were coded as being between -90 and $90 \mathrm{deg}$. RT, unless otherwise indicated, measures the duration from when the response meter appeared (key presses prior to this time were not accepted) until the up arrow key was pressed, which indicated that the subject's response was final.

Subjects were instructed to focus on accuracy but were also told that it might be easier to do the task if they responded while the stimulus was fresh in their minds. For the practice trials, we gave subjects visual feedback with a white line overlaid on their response. For test trials, the subjects were provided with auditory feedback after each test trial to encourage accuracy. A higher-pitched "ding" sound (www.freesound.org/people/HardPCM/sounds/32950/) indicated that they were below a fixed threshold (12 deg for the first two experiments, and $10 \mathrm{deg}$ for all others) or a negative, lower-pitched "donk" sound (www.freesound.org/people/ tombola/sounds/49219/) that indicated they were above the threshold. After the computer task, subjects filled out a selfreport questionnaire.

\section{Statistical analyses}

Statistical analyses used the $\mathrm{R}$ programming language ( $\mathrm{R}$ Development Core Team, 2011). To measure bias in the subjects' responses, we used the signed errors (range -90 to $90 \mathrm{deg}$, chance $=0 \mathrm{deg}$ ). For measuring the precision of the subjects' responses, we used the absolute value of the error (measured in degrees of angular deviation; range 0 $90 \mathrm{deg}$, chance $=45 \mathrm{deg}$ ). The unsigned error of subjects' responses yields a measure that is less sensitive to outliers, is similar to the measure used by Prinzmental et al. (1998; Prinzmetal et al., 1997), and is a superior measure for nonGaussian data (Gorard, 2005). Although these results are not reported, repeating the analyses with the standard deviation did not qualitatively alter the pattern of results.

As a summary statistic for each subject's RT responses we used the median value, since the distribution of RTs was skewed. (Separate analyses on the skew of the RT distributions showed no effect of condition.) Using median RTs decreased the sensitivity to outliers by decreasing the weight given to the slowest trials. While sample medians can overestimate population medians (Miller, 1988), our sample sizes per subject were large. Furthermore, since the cues were uninformative, the numbers of validly and invalidly cued trials were generally equal; thus, any overestimation would be equivalent between the conditions we compared. No subjects or trials were excluded from the analyses. Except where indicated, statistics were computed using analyses of variance. When post-hoc $t$ tests were performed, the significance value was adjusted for the number of comparisons using Bonferroni's correction.

\section{Results}

Experiments 1s, 2s, 1d, and 2d: Orientation judgments of static and dynamic stimuli with noninformative exogenous cuing and varying delays

The first set of experiments addressed the base question of whether the validity of a noninformative exogenous cue 
would affect the accuracy of an orientation judgment for either static (Exp. 1s; "s" for static) or dynamic (Exp. 1d; "d" for dynamic) stimuli. Table 1 shows the mean accuracy and RTs for both stimulus types and all cue conditions. We found that the accuracy of judgments of orientation for static patches was affected by cue validity $[F(2,38)=4.3, p=$ $.02]$ : Validly cued trials were reported more accurately than were invalidly cued trials $[t(19)=3.3, p=.01$, adjusted]. Valid cuing also decreased RTs $[F(2,38)=20.6, p<.001]$, with RTs decreasing from invalidly cued trials, to neutral trials, to validly cued trials $[t(19)>3.0, p<.03$, adjusted for all three comparisons]. This effect on RTs is particularly interesting as subjects were instructed to prioritize accuracy, and trial-by-trial feedback depended only on the accuracy of their responses.

Qualitatively similar results were found for the accuracy of judgments of orientation for the dynamic stimuli, but the effect on median accuracy with cuing was nonsignificant $[F$ $(2,38)=1.1, p=.33$; note, though, that similar analyses for subsequent experiments with the same stimuli and judgment are qualitatively similar and statistically significant]. RTs were affected by cue validity $[F(2,38)=20.9, p<.001]$, with faster RTs for validly cued than for neutral $[t(19)=4.8$, $p<.001$, adj.] or for invalidly cued $[t(19)=7.4, p<.001$, adj.] trials.

The effect of cue validity on orientation judgments was not due to changes in bias (which used signed errors, rather than the absolute values of the errors). The mean signed errors did not differ from zero [mean error (static stimuli) $=0.24 \mathrm{deg} ; t$ $(19)=1.0, p=.33$; mean error $($ dynamic stimuli $)=0.34 \mathrm{deg} ; t$ $(19)=1.4, \mathrm{~V} p=.18]$, and we found no interaction between cue validity and bias [with static stimuli, $F(2,38)=0.07$, $p=.93$; with dynamic stimuli, $F(2,38)=1.9, p=.16]$.

The group-level effects could also be seen at the level of individual performances (Fig. 2). The effect on RT was most consistent, with 19 of 20 subjects showing faster RTs for validly cued stimuli in both Experiments $1 \mathrm{~s}$ and $1 \mathrm{~d}$. The proportion of subjects showing smaller median errors for validly cued trials was 15 of 20 ( $p<.05$, binomial test) for both static and dynamic stimuli. The overall accuracy of the orientation judgments did not differ between the two experiments [mean (of median accuracy) $=8.6 \mathrm{deg}$ for static; mean $($ of median accuracy) $=9.0 \mathrm{deg}$ for dynamic; $t(32)=-0.5$, $p=.61$, Welch two-sample]. Likewise, there was no difference in RTs between these two experiments [static stimuli, mean (of

Table 1 Experiment 1: Cue validity effects on accuracy (in degrees) and response times (in milliseconds)

\begin{tabular}{llll}
\hline & Invalid & Neutral & Valid \\
\hline Orientation & $9.04 \mathrm{deg} / 825 \mathrm{~ms}$ & $8.51 \mathrm{deg} / 789 \mathrm{~ms}$ & $8.24 \mathrm{deg} / 762 \mathrm{~ms}$ \\
Motion & $9.17 \mathrm{deg} / 815 \mathrm{~ms}$ & $8.91 \mathrm{deg} / 802 \mathrm{~ms}$ & $8.88 \mathrm{deg} / 764 \mathrm{~ms}$ \\
\hline
\end{tabular}
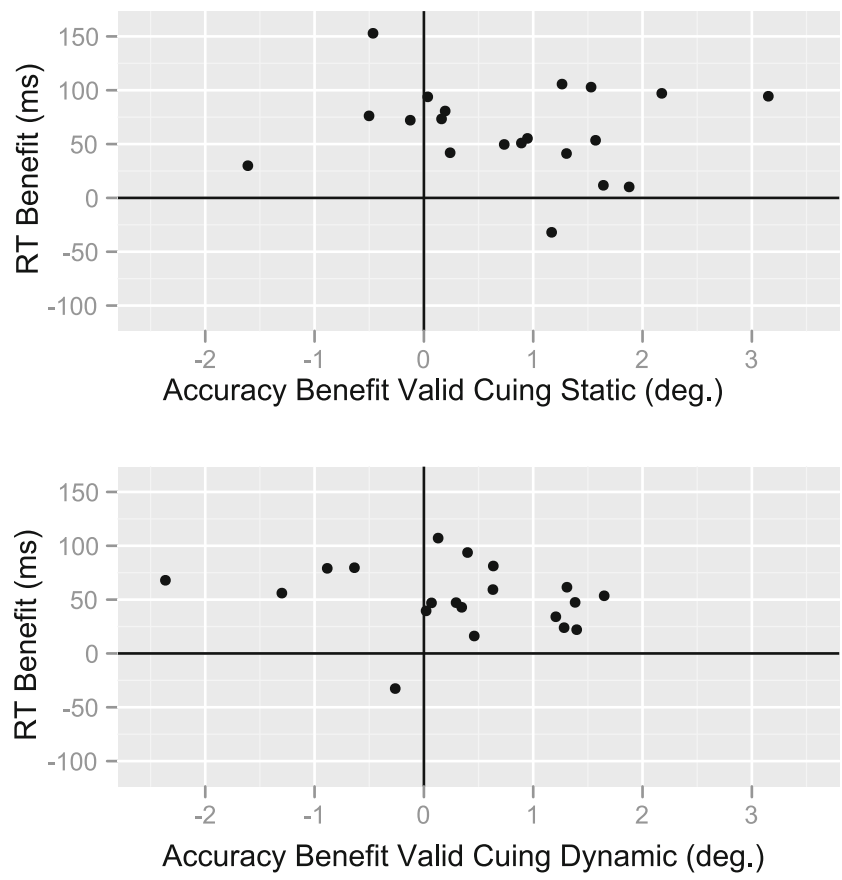

Fig. 2 Cue effects: Differences in RTs and accuracy due to cuing (Exps. 1s and 1d). Judgments of static stimuli are shown in the top panel, and those of dynamic stimuli on the bottom. In both experiments, 19/20 subjects decreased their RTs with valid cues, and 15/20 showed improved accuracy

medians $)=791 \mathrm{~ms}$; dynamic stimuli, mean (of medians $)=$ $794 \mathrm{~ms} ; t(38)=-0.03, p=.97$, Welch two-sample].

The improvement in the accuracy of orientation judgments was associated with more confident, less vacillating, responses. In our task, vacillation is literally the number of direction switches that subjects make when selecting their response - that is, the number of times that they switch from moving in one direction to the other. Subjects were less likely to change the direction of their responses when responding to validly cued stimuli $[F(2,38)=4.2, p=$ $.02]$. A larger proportion of invalidly cued trials had direction switches $(M=9.0 \%)$ than did validly cued trials $(M=$ $7.3 \%)[t(19)=3.6, p=.006$, adj.]. This same interaction was also found for the dynamic stimuli $[F(2,38)=12.3, p<$ .001 , where a larger proportion of invalidly cued trials had switches $(M=9.6 \%)$ than did neutrally cued trials $(M=$ $7.5 \%)[t(19)=3.5, p=.007$, adj. $]$ or validly cued trials $(M=$ $6.8 \%)[t(19)=4.5, p<.001$, adj.].

As a replication, and to see how much the results depended on the delay between when the stimulus was presented and when responses were allowed, we repeated the experiments (called $2 \mathrm{~s}$ and $2 \mathrm{~d}$ ) with three different delays between the time that the stimulus and cue disappeared and the time when a response could be made (the original 200-ms delay, as well as 400- and 600-ms delays). As compared to the 200-ms delay, the 400 -ms delay resulted in a $20-\mathrm{ms}$ increase in the time to initiate a response for 
static stimuli, and an increase of $16 \mathrm{~ms}$ for dynamic stimuli; for the 600-ms delay, the increased times to initiate a response were $107 \mathrm{~ms}$ for static stimuli and $176 \mathrm{~ms}$ for dynamic stimuli). Despite these lengthenings, the same effects on speed and accuracy were observed (Table 2). For both static $[F(1,19)=9.8, p=.006]$ and dynamic $[F$ $(1,19)=4.7, p=.04]$ stimuli, we found an effect of cue validity on the accuracy of orientation judgments. For neither stimulus type was there an effect of delay nor any interaction between cue validity and delay $[F(2,38)<1.3$, $p \mathrm{~s} \geq .30$ for all tests].

Experiments 3s and 3d: Orientation judgments of static and dynamic stimuli with noninformative exogenous cuing at different levels of stimulus quality

Motivated by the ideas that attention may be most useful when the stimuli are difficult to discriminate, we examined the effect of exogenous cuing when stimulus quality was degraded by lowering contrast (static gratings) or lowering motion coherence (dot stimuli). For the static stimuli, we binned $\log$ contrast into ten equal-sized bins. We found a main effect of cue validity $[F(1,19)=72.4, p<.001]$, an effect of stimulus contrast $[F(9,171)=28.3, p<.001]$, and an interaction $[F(9,171)=7.7, p<.001]$. The interaction reflected the growing magnitude of the benefit of valid cues for stimuli at the lower end of the contrast range (Fig. 3). For dynamic stimuli, coherence was binned into nine bins. Valid cuing led to more accurate judgments $[F(1,19)=4.9, p=$ $.04]$. There was also an effect of coherence $[F(8,152)=$ $62.6, p<.001]$, but there was no reliable interaction $[F(8$, $152)=1.2, p=.30]$. The pattern seen, a relatively uniform benefit across the coherence range (and anchored at both ends), appears qualitatively different from that seen with the static stimuli (Fig. 3). As the neural systems for processing static and dynamic stimuli have distinct components, the basis for the different patterns seen in Experiments $3 \mathrm{~s}$ and $3 \mathrm{~d}$ might be due to distinct effects at the level of the distinct neural systems. However, it has also been pointed out to us that the way in which we degraded discriminability could also explain the differences between experiments. In Experiment $3 \mathrm{~s}$, the discriminability was reduced by lowering contrast. This could invoke compensation through a mechanism of stimulus enhancement (Carrasco et al., 2004;
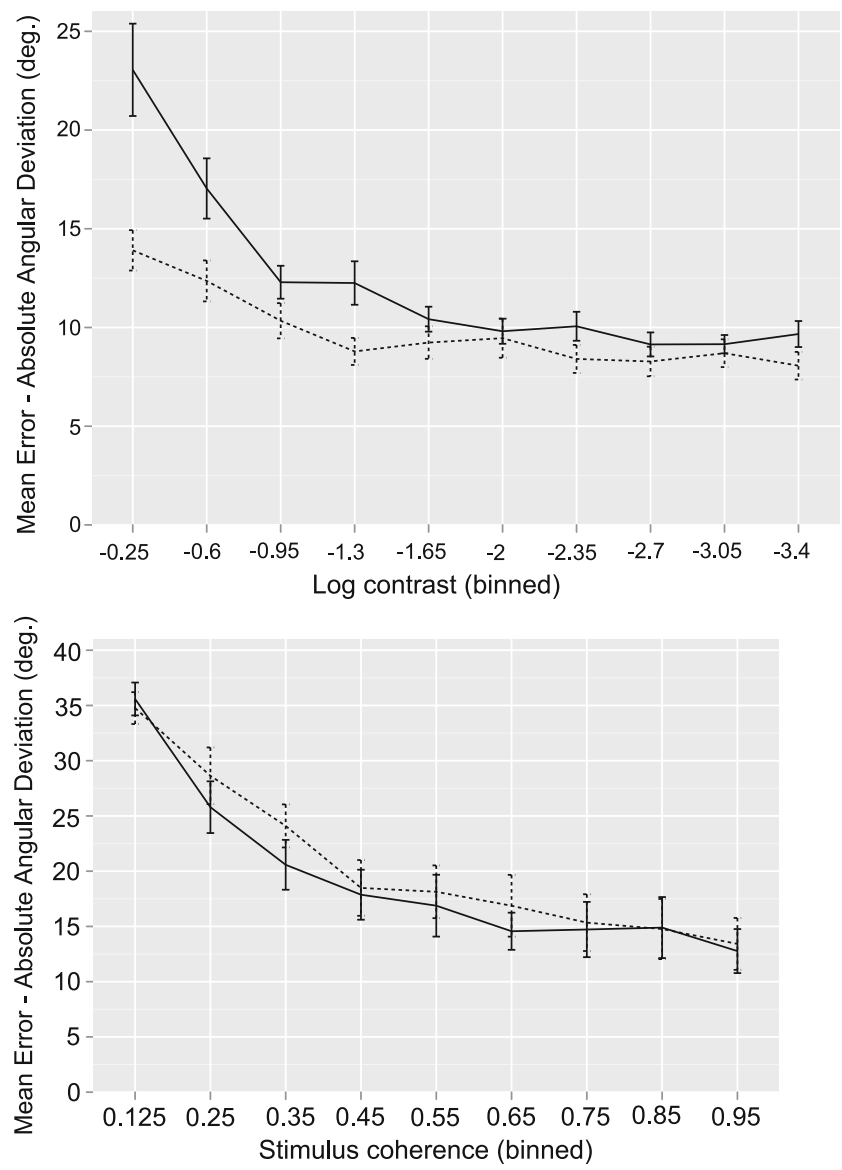

Fig. 3 Cuing benefits for orientation judgments at varying levels of contrast and stimulus coherence (Exps. 3d and 3s). (Top) The relative benefit of a valid cue (dotted line) for judging the orientation of a static grating increased as contrast decreased. (Bottom) For motion stimuli, valid cues increased the accuracy of motion judgments similarly across the spectrum of available coherence

Reynolds \& Chelazzi, 2004). In Experiment 3d, the discriminability was reduced by a combination of decreased signal intensity (fewer coherent dots) and an increase in noise (more incoherent dots). Thus, the difference in response to cuing could also reflect cuing effects on noise reduction (Dosher \& Lu, 2000a).

Responses to static and dynamic stimuli compared

We present the response distributions for our subjects in Experiments $1 \mathrm{~s}$ and $1 \mathrm{~d}$, in which there was no stimulus

Table 2 Experiment 2: Cue validity effects on accuracy (in degrees) and response times (in milliseconds) for trials of each delay

\begin{tabular}{|c|c|c|c|c|c|c|}
\hline & \multicolumn{2}{|l|}{$200 \mathrm{~ms}$} & \multicolumn{2}{|l|}{$400 \mathrm{~ms}$} & \multicolumn{2}{|l|}{$600 \mathrm{~ms}$} \\
\hline & Invalid & Valid & Invalid & Valid & Invalid & Valid \\
\hline Orientation & $9.85 \mathrm{deg} / 971 \mathrm{~ms}$ & $9.30 \mathrm{deg} / 913 \mathrm{~ms}$ & $9.75 \mathrm{deg} / 806 \mathrm{~ms}$ & $8.83 \mathrm{deg} / 768 \mathrm{~ms}$ & $9.70 \mathrm{deg} / 757 \mathrm{~ms}$ & $9.03 \mathrm{deg} / 729 \mathrm{~ms}$ \\
\hline Motion & $9.11 \mathrm{deg} / 958 \mathrm{~ms}$ & $8.56 \mathrm{deg} / 914 \mathrm{~ms}$ & $9.28 \mathrm{deg} / 794 \mathrm{~ms}$ & $8.64 \mathrm{deg} / 743 \mathrm{~ms}$ & $9.12 \mathrm{deg} / 705 \mathrm{~ms}$ & $9.16 \mathrm{deg} / 695 \mathrm{~ms}$ \\
\hline
\end{tabular}


degradation and the accuracies of responses were comparable (Fig. 4). Despite the uniform stimulus distribution in both experiments, subjects' response distributions are nonuniform. The data presented here are not about the accuracy
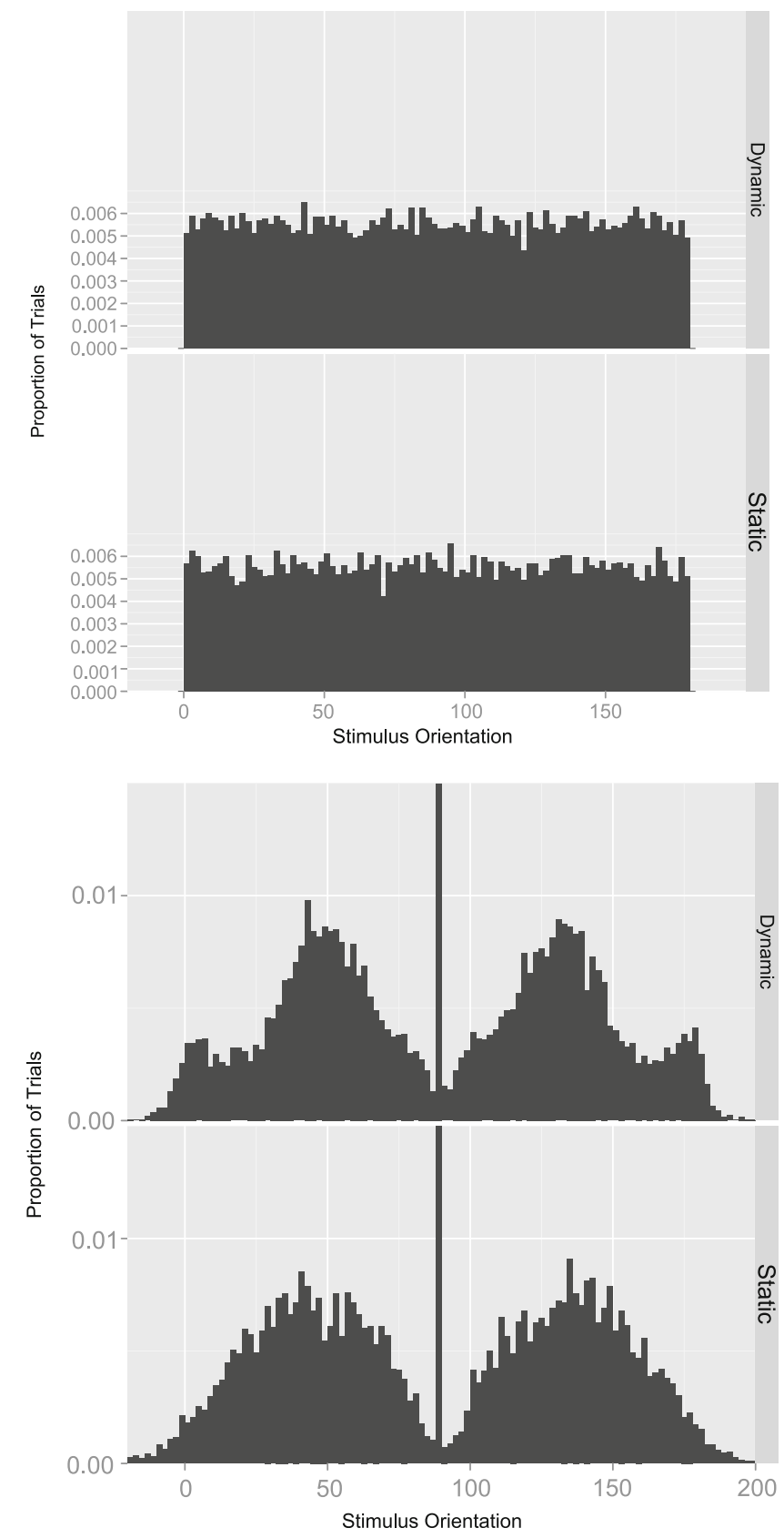

Fig. 4 Distributions of stimulus orientations and subject responses (Exps. 1d and 1s). On the top are the actual orientations, which are evenly distributed. On the bottom are the distributions of responses. For static stimuli, there is a bias to respond using the canonical oblique orientation ( $45 \mathrm{deg}$ from vertical), as well as with the default vertical orientation (the tall vertical bar in the middle of the lower plots is the data bar for the proportion of responses at $90 \mathrm{deg}$. This bar of the histogram is truncated by the scaling used to show the other angles). For dynamic stimuli, the pattern is similar, except that horizontal motion judgments are not shunned as responses, so that secondary peaks appear near the horizontal of judgments as a function of stimulus orientation (the "oblique effect"; Appelle, 1972), but demonstrate a bias to over report oblique orientations. In addition, the proportions of "horizontal" responses differ for judgments of dynamic and static stimuli, in that "horizontal" judgments are comparatively overrepreresented for dynamic stimuli. While the nature of the judgment and the statistics of the stimuli were identical, the response distributions were not. Thus, more than the nature of the cue and the type of judgment being made, stimulus motion, should be considered when evaluating the response properties.

\section{Discussion}

We have shown that a noninformative exogenous cue is associated with a decrease in the magnitude of errors when estimating the orientation of a static grating. Demonstrating that this effect is not an idiosyncrasy of one particular stimulus type, we showed that the same effect holds for judgments of the axis of motion of coherently moving dots. Unlike in prior related work, we did not need to mask the stimuli to observe an effect (Smith, Ratcliff, \& Wolfgang, 2004), nor did we need to use informative cues (Prinzmetal, McCool, \& Park, 2005; Prinzmetal, Park, \& Garrett, 2005). This report also describes a straightforward method for assessing the effect of exogenous attention on trial-by-trial measures of performance. It is similar to methods used by Prinzmetal et al. (1998; Prinzmetal et al., 1997) on dual-task effects. These procedures are all simply computer-aided elaborations of classical psychophysical methods.

Our demonstration that spatial attention has a consistent effect of improving a response variability measure of accuracy could be seen as being at variance with some prior reports. For example, Kerzel, Zarian, and Souto (2009) failed to find changes in a proportion correct measure of accuracy when stimuli were unmasked and a noninformative spatial cue was used. In that study, subjects made a judgment between two simultaneously presented stimuli. As was pointed out by Kerzel, Zarian, et al. (2010), this minimal visual search might have changed the task demands. In all of our experiments, we always presented a single stimulus, and that stimulus was always the target. Kerzel, Gauch, and Buetti (2010), however, failed to find an improved proportion correct with noninformative peripheral cues and a single stimulus. We believe that these different results reflect the different demands of classification and matching procedures. For making difficult discriminations, the most informative channels are those tuned off-channel; that is, discrimination is better when putting the most weight on neurons whose tuning curve peaks are not centered on either of the signals to be discriminated. Hol and Treue (2001) and Scolari and Serences (2010) have demonstrated such off- 
channel-tuning preferences when difficult perceptual classifications have to be made. With a reproduction paradigm, such as we used, the situation is different, and it may be optimal to "listen" to the population of neurons tuned for the stimulus to be reproduced.

The finding that noninformative spatial cues lead to better perceptual representations might at first seem inconsistent with selective-channel-weighting models of attention (Baldassi \& Verghese, 2005; Prinzmetal, McCool, \& Park, 2005). For example, Baldassi and Verghese had participants classify when a target appeared. The targets were oriented gratings and were cued with $100 \%$ valid cues to either location or orientation. The authors found that orientation cues showed particular benefits when the masks were highly similar to the target. The model that they developed interpreted the effect of valid orientation cuing as being mediated by the selective weighting of relevant orientation channels. The apparent inconsistency emerges because Baldassi and Verghese observed their effects for $100 \%$ valid orientation cues, and we observed ours for cues that were uninformative about both location and orientation. How would attentional networks know what orientation channels to weight in our paradigm? The paradox can be resolved, however, if we expand our ideas about what is a cue and what is a target. We assume that our noninformative spatial cue biased a particular screen location. On trials in which the cue was valid, the target appeared at that location. We can interpret this as a second, orientation cue, because this target/cue could lead to a subset of the orientation channels for that particular location being given greater weight. In this case, when the subjects in our task subsequently responded using what we call the response meter, they could also be viewed as solving a finely grained classification task. The subjects might end up having smaller deviations from veridical because the correct orientation for the matching task fell into an orientation "sweet spot" induced by what would conventionally be considered the task's target. This logic might also explain the difference between our results with a matching task and those of Kerzel and colleagues (e.g., Kerzel, Gauch, \& Buetti, 2010) using classification tasks. Our experimental procedures allowed subjects to benefit from the target as sensory prime; classification tasks do not typically afford this opportunity.

We also demonstrated that our method can be used to compare the effects of attention on different stimulus dimensions. Because our cue was a spatial cue, and because the static and dynamic versions of our experiments required similar judgments, one might predict that we would observe similar cue effects. As Experiments 3d and 3s demonstrated qualitatively different patterns of cue effects with stimulus degradation, as well as different distributions of subjects' responses, this revealed a heterogeneity for attentional consequences that cannot be explained in terms of stimulus statistics or task demands. Therefore, the effects of exogenous cuing cannot simply be some generic amplification that depends only on stimulus position and ignores stimulus characteristics.

\section{References}

Anton-Erxleben, K., Abrams, J., \& Carrasco, M. (2011). Equality judgments cannot distinguish between attention effects on appearance and criterion: A reply to Schneider (2011). Journal of Vision, 11(13):8, 1-8. doi:10.1167/11.13.8

Appelle, S. (1972). Perception and discrimination as a function of stimulus orientation: The "oblique effect" in man and animals. Psychological Bulletin, 78, 266-278.

Baldassi, S., \& Verghese, P. (2005). Attention to locations and features: Different top-down modulation of detector weights. Journal of Vision, 5(6), 7, 556-570. doi:10:1167/5.6.7

Carrasco, M., Ling, S., \& Read, S. (2004). Attention alters appearance. Nature Neuroscience, 7, 308-313. doi:10.1038/nn1194

Dosher, B. A., \& Lu, Z.-L. (2000a). Mechanisms of perceptual attention in precuing of location. Vision Research, 40, 1269-1292. doi:10.1016/S0042-6989(00)00019-5

Dosher, B. A., \& Lu, Z.-L. (2000b). Noise exclusion in spatial attention. Psychological Science, 11, 139-146. doi:10.1111/14679280.00229

Gorard, S. (2005). Revisiting a 90-year-old debate: The advantages of the mean deviation. British Journal of Educational Studies, 53, $417-430$.

Hol, K., \& Treue, S. (2001). Different populations of neurons contribute to the detection and discrimination of visual motion. Vision Research, 41, 685-689. doi:10.1016/S0042-6989(00)00314-X

Kerzel, D., Gauch, A., \& Buetti, S. (2010a). Involuntary attention with uncertainty: Peripheral cues improve perception of masked letters, but may impair perception of low-contrast letters. Journal of Vision, 10(12), 12, 1-13. doi:10:1167/10.12.12

Kerzel, D., Zarian, L., Gauch, A., \& Buetti, S. (2010b). Large effects of peripheral cues on appearance correlate with low precision. Journal of Vision, 10(11), 26, 1-14. doi:10:1167/10.11.26

Kerzel, D., Zarian, L., \& Souto, D. (2009). Involuntary cueing effects on accuracy measures: Stimulus and task dependence. Journal of Vision, 9(11), 16, 1-16. doi:10:1167/9.11.16

Lee, D., Itti, L., Koch, C., \& Braun, J. (1999). Attention activates winner-take-all competition among visual filters. Nature Neuroscience, 2, 375-381.

Miller, J. (1988). A warning about median reaction time. Journal of Experimental Psychology. Human Perception and Performance, 14, 539-543. doi:10.1037/0096-1523.14.3.539

Moray, N., Fitter, M., Ostry, D., Favreau, D., \& Nagy, V. (1976). Attention to pure tones. Quarterly Journal of Experimental Psychology, 28, 271-283.

Peirce, J. W. (2008). Generating stimuli for neuroscience using PsychoPy. Frontiers in Neuroinformatics, 2(10), 1-8. doi:10.3389/ neuro.11.010.2008

Posner, M. I., Snyder, C. R., \& Davidson, B. J. (1980). Attention and the detection of signals. Journal of Experimental Psychology. General, 109, 160-174. doi:10.1037/0096-3445.109.2.160

Prinzmetal, W., Amiri, H., Allen, K., \& Edwards, T. (1998). Phenomenology of attention: 1. Color, location, orientation, and spatial frequency. Perception, 24, 261-282.

Prinzmetal, W., McCool, C., \& Park, S. (2005a). Attention: Reaction time and accuracy reveal different mechanisms. Journal of Experimental Psychology. General, 134, 73-92. doi:10.1037/00963445.134.1.73 
Prinzmetal, W., Nwachuku, I., Bodanski, L., Blumenfeld, L., \& Shimizu, N. (1997). The phenomenology of attention: 2. Brightness and contrast. Consciousness and Cognition, 6, 372-412.

Prinzmetal, W., Park, S., \& Garrett, R. (2005b). Involuntary attention and identification accuracy. Perception \& Psychophysics, 67, 1344-1353. doi:10.3758/BF03193639

Prinzmetal, W., \& Wilson, A. (1997). The effect of attention on phenomenal length. Perception, 26, 193-205.

R Development Core Team (2011). R: A language and environment for statistical computing [Computer software environment]. Vienna, Austria: R Foundation for Statistical Computing. Available from www.R-project.org/
Reynolds, J. H., \& Chelazzi, L. (2004). Attentional modulation of visual processing. Annual Review of Neuroscience, 27, 611-647. doi:10.1146/annurev.neuro.26.041002.131039

Schneider, K. A. (2011). Attention alters decision criteria but not appearance: A reanalysis of Anton-Erxleben, Abrams, and Carrasco (2010). Journal of Vision, 11(13), 7, 1-8. doi:10.1167/11.13.7

Scolari, M., \& Serences, J. T. (2010). Basing perceptual decisions on the most informative sensory neurons. Journal of Neurophysiology, 104, 2266-2273. doi:10.1152/jn.00273.2010

Smith, P. L., Ratcliff, R., \& Wolfgang, B. J. (2004). Attention orienting and the time course of perceptual decisions: Response time distributions with masked and unmasked displays. Vision Research, 44, 1297-1320. doi:10.1016/j.visres.2004.01.002 